\title{
Self-consistent triaxial de Zeeuw-Carollo models
}

\author{
Parijat Thakur ${ }^{1}$, Ing-Guey Jiang ${ }^{1}$, M. Das ${ }^{2}$, D. K. Chakraborty ${ }^{3}$, and H. B. Ann ${ }^{2}$ \\ 1 Department of Physics and Institute of Astronomy, National Tsing-Hua University, Hsin-Chu 30013, Taiwan \\ e-mail: [pthakur; jiang]@phys.nthu.edu.tw \\ 2 Division of Science Education, Pusan National University, Busan 609-735, Korea \\ e-mail: [mdas; hbann]@pusan.ac. kr \\ 3 School of Studies in Physics, Pt. Ravishankar Shukla University, Raipur 492 010, India \\ e-mail: ircrsu@sancharnet.in
}

Received 6 May 2004 / Accepted 10 September 2007

ABSTRACT

\begin{abstract}
We use the standard method of Schwarzschild to construct self-consistent solutions for the triaxial de Zeeuw \& Carollo (1996) models with central density cusps. ZC96 models are triaxial generalizations of spherical $\gamma$-models of Dehnen whose densities vary as $r^{-\gamma}$ near the center and $r^{-4}$ at large radii and hence, possess a central density core for $\gamma=0$ and cusps for $\gamma>0$. We consider four triaxial models from ZC96, two prolate triaxials: $(p, q)=(0.65,0.60)$ with $\gamma=1.0$ and 1.5, and two oblate triaxials: $(p, q)=(0.95,0.60)$ with $\gamma=1.0$ and 1.5. We compute 4500 orbits in each model for time periods of $10^{5} T_{\mathrm{D}}$. We find that a large fraction of the orbits in each model are stochastic by means of their nonzero Liapunov exponents. The stochastic orbits in each model can sustain regular shapes for $\sim 10^{3} T_{\mathrm{D}}$ or longer, which suggests that they diffuse slowly through their allowed phase-space. With the exception of the oblate triaxial models with $\gamma=1.0$, our attempts to construct self-consistent solutions employing only the regular orbits fail for the remaining three models. However, the self-consistent solutions are found to exist for all models when the stochastic and regular orbits are treated in the same way because the mixing-time, $\sim 10^{4} T_{\mathrm{D}}$, is shorter than the integration time, $10^{5} T_{\mathrm{D}}$. Moreover, the "fully-mixed" solutions can also be constructed for all models when the stochastic orbits are fully mixed at 15 lowest energy shells. Thus, we conclude that the self-consistent solutions exist for our selected prolate and oblate triaxial models with $\gamma=1.0$ and 1.5 .
\end{abstract}

Key words. galaxies: kinematics and dynamics - galaxies: structure - methods: numerical

\section{Introduction}

It is natural to assume that elliptical galaxies can be triaxial, such as ellipsoids (Binney 1978). In this regard, Schwarzschild (1979) developed a method to explore the existence of triaxial ellipticals using a catalog of numerically integrated orbits, which eventually became a popular tool. The triaxial models he considered feature a central density core, and also show isophotal twists (Chakraborty \& Thakur 2000) representing the observed photometric properties of the majority of elliptical galaxies (Jedrzejewski 1987; Peletier et al. 1990). However, the ground-based (Moller et al. 1995) and Hubble Space Telescope (Crane et al. 1993; Jaffe et al. 1994; Ferrarese et al. 1994; Lauer et al. 1995; Faber et al. 1997) observations reveal that instead of central density cores, most of the elliptical galaxies have central density cusps. Thus, Merritt \& Fridman (1996) studied the effect of central density cusps on triaxial configurations but their models do not show isophotal twists. In their study, it was found that a "fully-mixed" solution exists for a maximally triaxial model with a weak-cusp. The aim of this paper is to construct self-consistent solutions for more realistic triaxial models in the sense of representing both of the above-mentioned significant observed properties of elliptical galaxies, namely central density cusps and isophotal twists. Here we used the triaxial models given by de Zeeuw \& Carollo (1996; hereafter ZC96), which have a central density core for $\gamma=0$ and a cusp for $\gamma>0$, and also show isophotal twists in their projection along the line-ofsight. Furthermore, it is worth mentioning that the ZC96 triaxial models are found to be useful in constraining the intrinsic shapes of elliptical galaxies using their projected properties (Thakur \& Chakraborty 2001).

The remainder of this paper is organized as follows. In Sect. 2, we present the ZC96 triaxial models. Section 3 describes equations of motion for the ZC96 triaxial models. Section 4 presents the integration of orbits. Section 5 deals with the computation of the Liapunov exponents. The method to construct the self-consistent triaxial models, is presented in Sect. 6 and Sect. 7 is devoted to the results and discussion.

\section{Triaxial de Zeeuw-Carollo models}

We have used the triaxial potentials of ZC96, given as

$V(r, \theta, \phi)=u(r)-v(r) Y_{2}^{\mathrm{o}}(\theta)+w(r) Y_{2}^{2}(\theta, \phi)$,

$V(x, y, z)=u(r)-v(r)\left(2 z^{2}-x^{2}-y^{2}\right) / 2 r^{2}+w(r) 3\left(x^{2}-y^{2}\right) / r^{2}$

where $(r, \theta, \phi)$ are spherical coordinates defined such that $x=$ $r \sin \theta \cos \phi, y=r \sin \theta \sin \phi$ and $z=r \cos \theta$, the functions $Y_{2}^{\mathrm{o}}(\theta)=\frac{3}{2} \cos ^{2} \theta-\frac{1}{2}$ and $Y_{2}^{2}(\theta, \phi)=3 \sin ^{2} \theta \cos 2 \phi$ are usual spherical harmonics, and $u(r), v(r)$ and $w(r)$ are three radial functions. Here $u(r)$ is chosen to be the potential of the spherical $\gamma$-models of Dehnen (1993), defined by

$u(r)= \begin{cases}\frac{G M}{r_{\mathrm{o}}} \ln \frac{r}{r+r_{\mathrm{o}}}, & \text { for } \gamma=2, \\ \frac{G M}{(2-\gamma) r_{\mathrm{o}}}\left[\left(\frac{r}{r+r_{\mathrm{o}}}\right)^{2-\gamma}-1\right], & \text { for } \gamma \neq 2,\end{cases}$

where $M$ is the total mass of the model, $r_{\mathrm{o}}$ is the scale-length and cusp parameter $\gamma$ can have a value in between $0 \leq \gamma<3$. 
Table 1. Model parameters and Liapunov time $\left(\tau_{\mathrm{L}}\right)$.

\begin{tabular}{ccccc|c}
\hline \hline Model & $p$ & $q$ & $\gamma$ & $\tau_{\mathrm{L}}$ (in units of $\left.T_{\mathrm{D}}\right)$ & Comments \\
\hline \multirow{2}{*}{ Models PT } & 0.65 & 0.60 & 1.0 & $\sim 21 \pm 0.46$ & \\
& 0.65 & 0.60 & 1.5 & $\sim 13 \pm 0.33$ & \\
\hline \multirow{2}{*}{ Models OT } & 0.95 & 0.60 & 1.0 & $\sim 25 \pm 0.50$ & Oblate Triaxial \\
& 0.95 & 0.60 & 1.5 & $\sim 17 \pm 0.40$ & \\
\hline
\end{tabular}

Furthermore, the functions $v(r)$ and $w(r)$ are considered as follows:

$v(r)=-\frac{G M r_{1} r^{2-\gamma}}{\left(r+r_{2}\right)^{4-\gamma}}, \quad w(r)=-\frac{G M r_{3} r^{2-\gamma}}{\left(r+r_{4}\right)^{4-\gamma}}$,

where $r_{1}, \ldots, r_{4}$ are constants.

The associated density distribution $\rho(r, \theta, \phi)$ follows from Poisson's equation

$\rho(r, \theta, \phi)=f(r)-g(r) Y_{2}^{\mathrm{o}}(\theta)+h(r) Y_{2}^{2}(\theta, \phi)$,

$\rho(x, y, z)=f(r)-g(r)\left(2 z^{2}-x^{2}-y^{2}\right) / 2 r^{2}+h(r) 3\left(x^{2}-y^{2}\right) / r^{2}$,

where $f(r), g(r)$, and $h(r)$ are taken from Eq. (2.5) of ZC96. The four ratios $r_{1} / r_{0}, \ldots, r_{4} / r_{0}$ can be expressed in terms of $\gamma$, and of the axial ratios of the density distribution at small and at large radii, denoted by $\left(p_{\mathrm{o}}, q_{\mathrm{o}}\right)$ and $\left(p_{\infty}, q_{\infty}\right)$ respectively, where the surfaces of constant density are approximately ellipsoidal, i.e., $\rho \sim \rho\left(m^{2}\right)$ with $m^{2}=x^{2}+y^{2} / p^{2}+z^{2} / q^{2}$. ZC96 models have a central density core for $\gamma=0$, while they have cusps in which the density diverges as $r^{-\gamma}$ at small radii for $\gamma>0$. On the other hand, the density falls off as $r^{-4}$ at large radii.

In this paper, we fix the values of axial ratios as $p_{\mathrm{o}}=p_{\infty} \equiv p$ and $q_{\mathrm{o}}=q_{\infty} \equiv q$ for all the calculations.

\section{Equation of motion for triaxial de Zeeuw-Carollo models}

The equation of motion is given by

$\frac{\mathrm{d}^{2} \boldsymbol{r}}{\mathrm{d} t^{2}}=\boldsymbol{F}=-\nabla V(x, y, z)$

where $\boldsymbol{F}$ is the force per unit mass, and $V(x, y, z)$ represents the triaxial potentials of ZC96 given in Eq. (1).

The force components $\left(F_{x}, F_{y}, F_{z}\right)=\left(F_{1}, F_{2}, F_{3}\right)$ representing the equations of motion by three scalar equations in the Cartesian coordinates, needed at each step of an orbit integration, can be calculated by the partial derivatives of $V(x, y, z)$ with respect to $(x, y, z)=\left(x_{1}, x_{2}, x_{3}\right)$. These can be written as

$$
\begin{aligned}
F_{i}= & -\frac{x_{i}}{r}\left[u_{\mathrm{D}}-\left(\frac{v_{\mathrm{D}}}{2 r^{2}}-\frac{v_{r}}{r^{3}}\right)\left(2 x_{3}^{2}-x_{1}^{2}-x_{2}^{2}\right)+A_{1} \frac{v_{r}}{r}\right. \\
& \left.+A_{2}\left(\frac{w_{\mathrm{D}}}{r^{2}}-\frac{2 w_{r}}{r^{3}}\right)\left(x_{1}^{2}-x_{2}^{2}\right)+A_{3} \frac{w_{r}}{r}\right],
\end{aligned}
$$

where $\left(A_{1}, A_{2}, A_{3}\right)$ are equal to $(1,3,6),(1,3,-6)$, and $(-2,3,0)$ for $i=1,2,3$, respectively. Furthermore,

$$
\begin{aligned}
& u_{\mathrm{D}}=\left(\frac{r}{r+1}\right)^{1-\gamma}\left[\frac{1}{r+1}-\frac{r}{(r+1)^{2}}\right], \\
& v_{\mathrm{D}}=-r_{1}\left[\frac{(2-\gamma) r^{1-\gamma}}{\left(r+r_{2}\right)^{4-\gamma}}-\frac{(4-\gamma) r^{2-\gamma}}{\left(r+r_{2}\right)^{5-\gamma}}\right], \\
& w_{\mathrm{D}}=-r_{3}\left[\frac{(2-\gamma) r^{1-\gamma}}{\left(r+r_{4}\right)^{4-\gamma}}-\frac{(4-\gamma) r^{2-\gamma}}{\left(r+r_{4}\right)^{5-\gamma}}\right]
\end{aligned}
$$

are the derivatives of $u_{r}, v_{r}$, and $w_{r}$ with respect to $r$, respectively. Here the expressions of $u_{r}, v_{r}$, and $w_{r}$ can be derived from those of $u(r), v(r)$, and $w(r)$ given in Eqs. (2), (3) using $G=M=r_{\mathrm{o}}=$ 1 , respectively.

\section{Integration of orbits}

To integrate orbits, we have considered four triaxial models of ZC96, which are listed in Table 1. Here the first two models are prolate triaxials: $(p, q)=(0.65,0.60)$ with $\gamma=1.0$ and 1.5 , while the remaining two are oblate triaxials: $(p, q)=(0.95,0.60)$ with $\gamma=1.0$ and 1.5. In this paper, the former models will be called Models PT, whereas the latter models will be referred to as Models OT.

Since the triaxial mass models of ZC96 are very centrally concentrated, the numerical algorithm required for integrating the orbits must be extremely accurate and flexible. We have used a FORTRAN routine, RK78, which was kindly made available by Prof. D. Pfenniger. This routine follows the 7/8 order Runga-Kutta algorithm described in Fehlberg (1968), which incorporates a variable time step in order to maintain a specified accuracy from one integration step to the next. The accuracy parameter REPS in all the integrations is chosen to be $10^{-8}$. Energy is typically conserved to a few parts in $10^{9}$ over $10^{5}$ dynamical times with this choice of REPS.

For each of the four selected triaxial models, we followed the scheme developed by Schwarzschild (1993) in assigning initial conditions from the $x-z$ start-space with $v_{x}=v_{z}=0$ and stationary (equipotential) start-space with $v_{x}=v_{y}=v_{z}=0$. As in Merritt \& Fridman (1996), the orbits in both start-spaces are assigned a value from a set of 20 energies, defined as the values of the potential on the $x$-axis of a set of 20 ellipsoidal shells. The radius, energy and dynamical time $\left(T_{\mathrm{D}}\right)$ of each ellipsoidal shell are given in Table 2 for all models. Here energy dependent "dynamical time" $T_{\mathrm{D}}$ is defined as the period of the 1:1 resonant $x$ - $y$ periodic orbit. For the $x-z$ start-space, a total of 150 starting points per shell are calculated for all models. Moreover, the stationary start-space grid is defined as in Schwarzschild (1993). However, as opposed to 64 starting points in Merritt \& Fridman (1996), only 25 starting points are calculated in each of the three sectors on an equipotential octant, resulting in a total of 75 starting points per shell in the stationary start-space. Here, as the Liapunov exponents are computed for long periods of $10^{5} T_{\mathrm{D}}$ (see Sect. 5), we reduce the number of starting points to save computing time. Thus, a total of 4500 starting points are calculated for each of the selected models due to their division into a set of 20 ellipsoidal shells. For each of these starting points, we have integrated the orbit over a time interval of $10^{5} T_{\mathrm{D}}$.

\section{Computation of Liapunov exponents}

For detecting and quantifying stochasticity, we have followed Merritt \& Fridman (1996) and computed approximations to 
Table 2. Shell parameters for Models PT and OT.

\begin{tabular}{|c|c|c|c|c|c|c|c|c|c|c|}
\hline \multirow[t]{2}{*}{ Shell } & \multicolumn{2}{|c|}{${\underline{\text { Radius }^{a}}}^{a}$} & \multicolumn{2}{|c|}{ Energy for Models PT } & \multicolumn{2}{|c|}{$T_{\mathrm{D}}$ for Models PT } & \multicolumn{2}{|c|}{ Energy for Models OT } & \multicolumn{2}{|c|}{$T_{\mathrm{D}}$ for Models OT } \\
\hline & $\gamma=1.0$ & $\gamma=1.5$ & $\gamma=1.0$ & $\gamma=1.5$ & $\gamma=1.0$ & $\gamma=1.5$ & $\gamma=1.0$ & $\gamma=1.5$ & $\gamma=1.0$ & $\gamma=1.5$ \\
\hline 1 & 0.2791 & 0.1512 & -0.8103 & -1.3203 & 2.916 & 1.059 & -0.7963 & -1.2974 & 3.204 & 1.156 \\
\hline 2 & 0.4464 & 0.2635 & -0.7265 & -1.1378 & 3.980 & 1.668 & -0.7089 & -1.1116 & 4.356 & 1.812 \\
\hline 3 & 0.6076 & 0.3760 & -0.6600 & -1.0073 & 4.956 & 2.260 & -0.6407 & -0.9800 & 5.412 & 2.452 \\
\hline 4 & 0.7744 & 0.4949 & -0.6023 & -0.9017 & 5.964 & 2.876 & -0.5824 & -0.8744 & 6.492 & 3.116 \\
\hline 5 & 0.9530 & 0.6238 & -0.5503 & -0.8112 & 7.044 & 3.548 & -0.5304 & -0.7845 & 7.644 & 3.836 \\
\hline 6 & 1.1483 & 0.7662 & -0.5024 & -0.7310 & 8.244 & 4.299 & -0.4830 & -0.7054 & 8.916 & 4.636 \\
\hline 7 & 1.3660 & 0.9259 & -0.4575 & -0.6584 & 9.604 & 5.156 & -0.4390 & -0.6343 & 10.357 & 5.548 \\
\hline 8 & 1.6124 & 1.1075 & -0.4151 & -0.5920 & 11.173 & 6.156 & -0.3978 & -0.5694 & 12.013 & 6.604 \\
\hline 9 & 1.8956 & 1.3172 & -0.3747 & -0.5298 & 13.030 & 7.332 & -0.3589 & -0.5094 & 13.958 & 7.852 \\
\hline 10 & 2.2265 & 1.5628 & -0.3362 & -0.4718 & 15.263 & 8.764 & -0.3220 & -0.4535 & 16.303 & 9.356 \\
\hline 11 & 2.6199 & 1.8555 & -0.2993 & -0.4171 & 18.014 & 10.533 & -0.2867 & -0.4011 & 19.181 & 11.205 \\
\hline 12 & 3.0972 & 2.2114 & -0.2637 & -0.3654 & 21.499 & 12.774 & -0.2529 & -0.3516 & 22.803 & 13.542 \\
\hline 13 & 3.6903 & 2.6544 & -0.2296 & -0.3164 & 26.033 & 15.703 & -0.2205 & -0.3048 & 27.505 & 16.575 \\
\hline 14 & 4.4495 & 3.2220 & -0.1967 & -0.2697 & 32.174 & 19.661 & -0.1893 & -0.2603 & 33.845 & 20.668 \\
\hline 15 & 5.4580 & 3.9767 & -0.1650 & -0.2252 & 40.877 & 25.282 & -0.1593 & -0.2179 & 42.800 & 26.457 \\
\hline 16 & 6.8661 & 5.0312 & -0.1345 & -0.1829 & 54.026 & 33.781 & -0.1304 & -0.1775 & 56.265 & 35.164 \\
\hline 17 & 8.9736 & 6.6103 & -0.1052 & -0.1424 & 75.726 & 47.805 & -0.1024 & -0.1388 & 78.397 & 49.468 \\
\hline 18 & 12.4807 & 9.2393 & -0.0771 & -0.1040 & 116.652 & 74.287 & -0.0754 & -0.1018 & 119.946 & 76.358 \\
\hline 19 & 19.4875 & 14.4930 & -0.0502 & -0.0674 & 214.727 & 137.42 & -0.0494 & -0.0664 & 219.139 & 140.18 \\
\hline 20 & 40.4939 & 30.2466 & -0.0245 & -0.0327 & 605.260 & 392.30 & -0.0243 & -0.0325 & 611.661 & 396.64 \\
\hline
\end{tabular}

${ }^{a}$ On $x$-axis.

the six Liapunov exponents, which can be ordered by size as $\sigma_{1} \geq \sigma_{2} \geq \ldots \geq \sigma_{6}$, by integrating each of 4500 orbits for long periods of $10^{5} T_{\mathrm{D}}$ using the Gram-Schmidt orthogonalization technique described by Benettin et al. (1980). To perform this technique, we have used a FORTRAN routine, LIAMAG, which was kindly made available by Prof. D. Pfenniger from the Geneva Observatory group. In this routine, the second derivatives of the potential with respect to position are required for determination of the evolution of the perturbed orbits (Udry \& Pfenniger 1988), which are given in Appendix A.

As in Merritt \& Fridman (1996), we have restricted our attention to the three positive Liapunov exponents, since $\sigma_{i}=-\sigma_{7-i}$ with $i=1,2,3$. For all models, we have calculated the Liapunov times $\left(\tau_{\mathrm{L}}\right)$, corresponding to the instability timescale between neighboring orbits, which are found to be approximately the same for all energy shells when they are scaled in dynamical times $\left(T_{\mathrm{D}}\right)$. Table 1 gives these calculated values of Liapunov time $\left(\tau_{\mathrm{L}}\right)$ in units of $T_{\mathrm{D}}$ for all models. Here Models PT with $\gamma=$ 1.5 appears as the most stochastic, since its Liapunov time $\left(\tau_{\mathrm{L}}\right)$ is smaller compared to other selected models. The sum of all three positive Liapunov exponents is defined as "Kolmogorov entropy" $\left(h_{k}=\sum_{i=1,3} \sigma_{i}\right)$. In order to distinguish regular from stochastic orbits for all models, and unlike the case of Poon \& Merritt (2004) who use the histogram of $\sigma_{1} T_{\mathrm{D}}$, we consider the histogram of $h_{k} T_{\mathrm{D}}$ for the orbits at each energy shell from both start-spaces. Since the histogram of $h_{k} T_{\mathrm{D}}$ is found to have similar behaviour for all models, we present it in Fig. 1 for the orbits at shell 13 from the stationary start-space of the most stochastic model (i.e., Models PT with $\gamma=1.5$ ). As can be seen from Fig. 1, the separation of orbits into two groups representing two peaks becomes apparent as the integration time increases. Out of these two peaks, one is situated at narrow regions near zero showing the regular orbits, whereas the other is located at a non-zero value with larger spread that decreases with the integration time, representing the stochastic orbits. After a time interval of $\sim 10^{3} T_{\mathrm{D}}, h_{k} T_{\mathrm{D}}$ of the stochastic orbits at each energy shell for all models are found to approach common values, and their mean values do not change very much. This suggests the

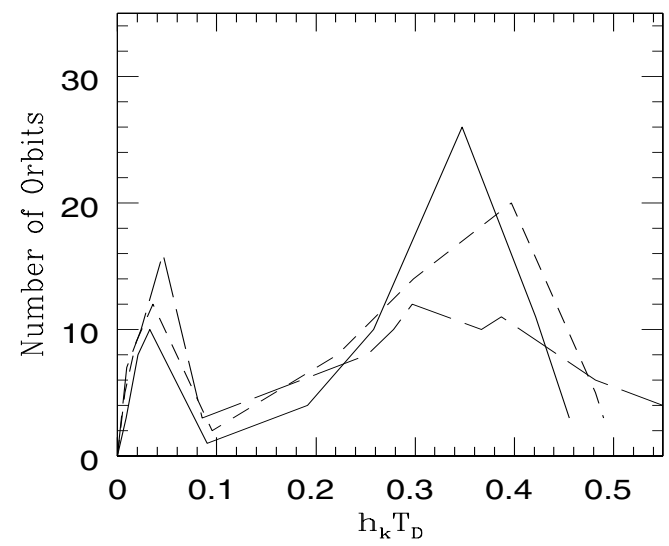

Fig. 1. Histogram of $h_{\mathrm{k}} T_{\mathrm{D}}$ for the orbits at shell 13 from the stationary start-space of Models PT with $\gamma=1.5$, which is the most stochastic model. Long-dash line: $t=10^{3} T_{\mathrm{D}}$; short dash line: $t=10^{4} T_{\mathrm{D}}$; solid line: $t=10^{5} T_{\mathrm{D}}$.

following: (1) the stochastic orbits in all models diffuse slowly through their allowed phase-space, and can sustain their regular shapes for $\sim 10^{3} T_{\mathrm{D}}$ or longer; (2) the mixing-time associated with diffusion through the Arnold web would be $\sim 10^{4} T_{\mathrm{D}}$ (cf. Merritt \& Valluri 1996), since it is clear from Fig. 1 that there is still a lot of mixing occurring at $\sim 10^{3} T_{\mathrm{D}}$. Our computation of the Liapunov exponents for periods of $10^{5} T_{\mathrm{D}}$, which is two orders of magnitude longer than others in the literature, allows us to achieve these results.

At the integration time of $10^{5} T_{\mathrm{D}}$, the critical value, $h_{\mathrm{kc}} T_{\mathrm{D}} \approx$ $10^{-1.03}$, is found to separate the orbits into two peaks in the histograms of $h_{k} T_{\mathrm{D}}$ at each energy shell from both start-spaces for all models. Thus, the orbits are considered to be stochastic if $h_{k} T_{\mathrm{D}}>h_{\mathrm{kc}} T_{\mathrm{D}}$. As can be seen from Fig. 2, a large fraction of the orbits at all energy shells from the stationary start-space are found to be stochastic for all models. For each of the two selected values of $\gamma$, a larger fraction of the stochastic orbits are found in Models PT than in Models OT. Furthermore, Models PT (Models OT) with $\gamma=1.5$ have a relatively larger fraction of 

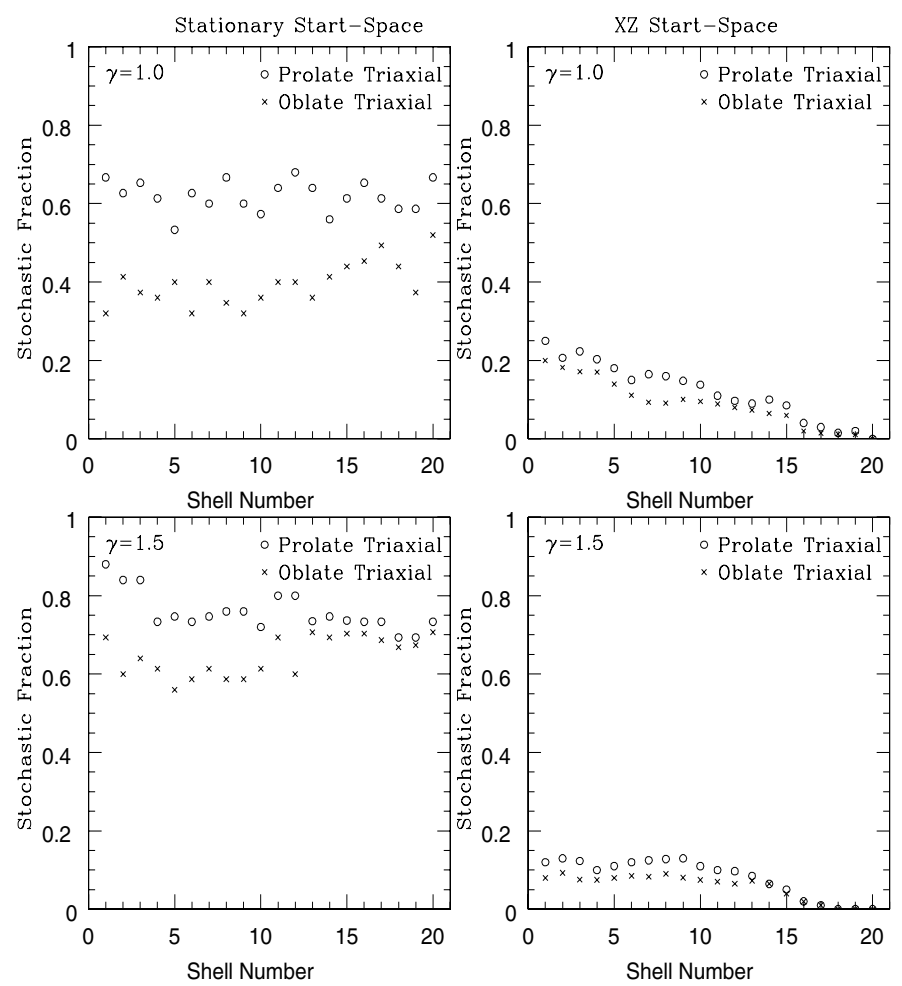

Fig. 2. Fraction of the stochastic orbits per shell in the orbit libraries. The left panels are for the stationary start-space, whereas the right ones are for the $x-z$ start-space. The cusp parameter $\gamma$ is given in the top left corner of each panel. Circles: models PT; crosses: Models OT.

the stochastic orbits than those of Models PT (Models OT) with $\gamma=1.0$. On the other hand, a significant fraction of the orbits from the $x-z$ start-space at each energy shell are also found to be stochastic for all models.

\section{Construction of self-consistent models}

Self-consistent equilibrium models can be constructed using the standard method of Schwarzschild (1979). Here a linear superposition of orbits is sought, each populated with an appropriate number of stars that could reproduce the mass of each cell. This method is formulated as

$$
\begin{array}{r}
\sum_{i=1}^{M} C(i) B(i, j)=D(j)(j=1 \ldots, N) \quad \text { with } \\
C(i) \geq 0, \quad(i=1, \ldots, M),
\end{array}
$$

where $B(i, j)$ is the time spent by the $i$ th orbit in the $j$ th cell, $D(j)$ is the mass of the $j$ th cell, and $C(i)$ is a weight associated with the $i$ th orbit, also called the non-negative occupation number of that orbit. To employ Schwarzschild's method, all models are divided into 960 cells by following the scheme set by Merritt \& Fridman (1996). Furthermore, as in Schwarzschild (1993), the masses of 960 cells and the time spent by each orbit in 960 cells are normalized to unity (i.e., $\sum_{j=1}^{960} D(j)=1$ and $\left.\sum_{j=1}^{960} B(i, j)=1\right)$. Using Lucy's (1974) iterations method as formulated in Schwarzschild (1993), we have then minimized the mean square deviation in the cell masses, i.e.,

$\chi^{2}=\frac{1}{N} \sum_{j=1}^{N}\left(D(j)-\sum_{i=1}^{M} C(i) B(i, j)\right)^{2}$, where $N=960$ and $M=$ total number of supplied orbits. In this paper, we have used the departure from selfconsistency to present our results, which is defined as $\delta=$ $\left(\sqrt{\chi^{2}} /\right.$ average mass per cell) (cf. Merritt \& Fridman 1996). In order to start the Lucy iterations, we first chose the trial values of $C(i)=$ const. Later, in each iteration, we compute $C(i)_{\text {new }}$ to derive $\delta$. We continue the iterations until satisfactory convergence in $\delta$ is achieved, which is found to be around the $\sim 30000$ iterations for all models. We repeat this procedure for an ever-larger, randomly selected sample of orbits and then $\delta$ as a function of the number of orbits is plotted. If a self-consistent solution exists, $\delta$ would decrease rapidly with the number of orbits.

\section{Results and discussion}

Our result in Sect. 5 supports previous findings (Merritt \& Fridman 1996; Wachlin \& Ferraz-Mello 1998; Siopis \& Kandrup 2000; Kandrup \& Siopis 2003; Poon \& Merritt 2004) that a large fraction of the orbits from the stationary start-space for models with $\gamma>0.0$ are stochastic, while there is a significant fraction of the stochastic orbits in the $x-z$ start-space as well. Furthermore, a number of authors (Merritt \& Fridman 1996; Holley-Bockelmann et al. 2001, 2002; Poon \& Merritt 2004) have claimed that cuspy triaxial equilibria can be constructed with sizable fractions of the stochastic orbits. This encouraged us to employ the stochastic orbits along with regular orbits while constructing the self-consistent solutions for all models listed in Table 1 . In order to carry out this, we followed the method given in Sect. 6, and the results are presented in Fig. 3. Since the mixing-time, $\sim 10^{4} T_{\mathrm{D}}$, is shorter than the integration time, $10^{5} T_{\mathrm{D}}$, we attempt to construct self-consistent solutions in which the stochastic and regular orbits are treated in the same way, allowing each stochastic orbit to have an arbitrary occupation number. Thus, a total of 4500 orbits are employed and then $\delta$ as a function of the number of orbits are plotted with "circles" in Fig. 3. Here $\delta$ decreases very quickly with the number of orbits and converges well for all models. Therefore, we conclude that the self-consistent solutions are found to exist for all models considered in this paper.

Furthermore, we performed an experiment to construct the self-consistent solutions utilizing only the regular orbits, although there is no obvious physical reason why nature would host only the regular orbits. The results of this study are represented by "crosses" in Fig. 3. Except for Models OT with $\gamma=1.0$, the remaining three models do not show better convergence in $\delta$ with increasing number of provided orbits. So, we conclude that the regular orbits can provide a sufficient variety of shapes to construct the self-consistent solution for Models OT with $\gamma=1.0$, which has a larger fraction of regular orbits than other selected models (see Fig. 2).

Although we have already shown the existence of the selfconsistent solutions by "circles" in Fig. 3, it would still be interesting to construct the "fully-mixed" solutions for all models due to the reasons listed in Merritt \& Fridman (1996). Thus, we attempted to construct the "fully-mixed" solutions by following Merritt \& Fridman (1996). The results are shown in Fig. 3 by "square" and "star" when the stochastic orbits are "fully-mixed" at 10 and 15 lowest energy shells, respectively. For each of these two cases, the convergence in $\delta$ is found to be good, which suggests that the "fully-mixed" solutions can be constructed for all models. Thus, we conclude that the self-consistent solutions exist for all models considered in this paper. 

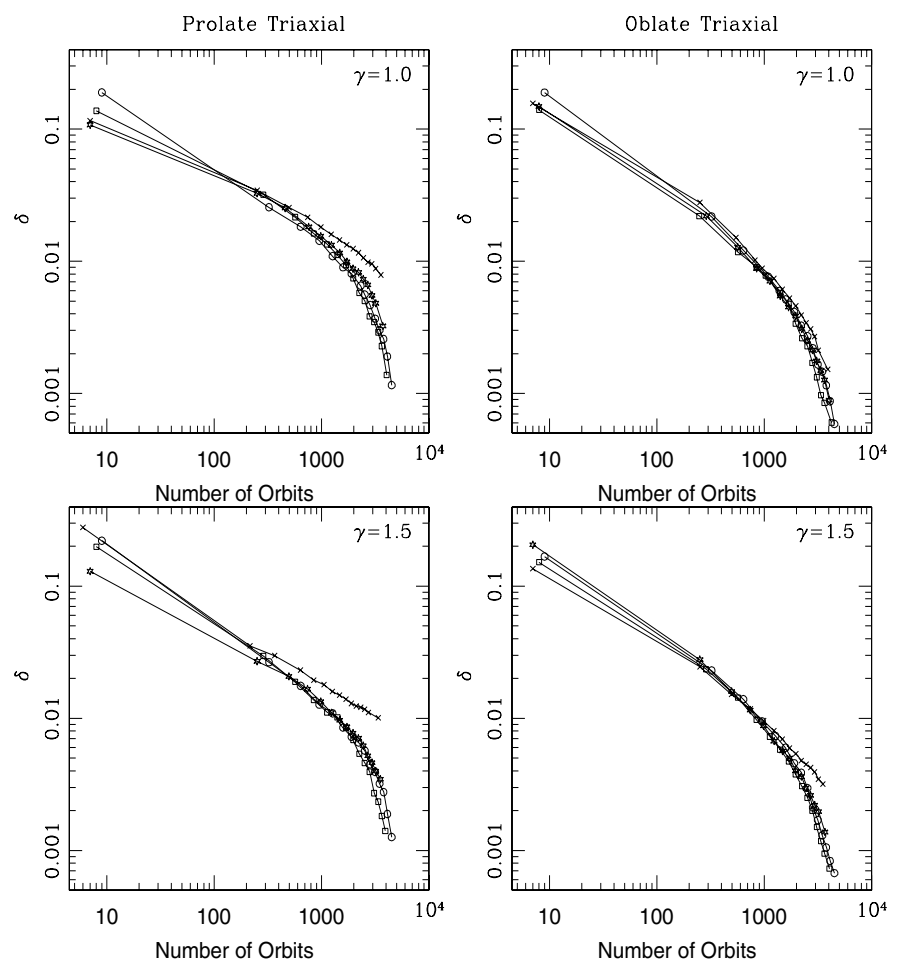

Fig. 3. Departure from self-consistency $(\delta)$ as a function of the number of orbits supplied to the minimization routine. Left panels: models PT with $\gamma=(1.0,1.5)$; right panels: models OT with $\gamma=(1.0,1.5)$. Circles: all orbits; crosses: regular orbits only; squares: the stochastic orbits are fully mixed at 10 lowest energy shells; stars: the stochastic orbits are fully mixed at 15 lowest energy shells.

Acknowledgements. We thank the anonymous referee for useful remarks and suggestions that improved the present paper enormously. The computer programs for integrating orbits and computing Liapunov exponents were written by the Geneva Observatory Group and kindly made available to us by Prof. D. Pfenniger. The computations were done by PC Cluster located at Department of Physics and Institute of Astronomy, National Tsing-Hua University, Hsinchu, Taiwan. P.T. would like to express his sincere thanks to National Science Council (NSC), Taiwan, for granting postdoctoral fellowship through grant: NSC 96-2811-M-007-006. P.T. and H.B. are also thankful to ARCSEC for providing support. This study was also financially supported by Pusan National University in the program, Post-Doc 2004.

\section{Appendix A: The force derivatives}

Here we present the derivatives of the gravitational forces corresponding to the ZC96 models, which are calculated by the gradient of the three components of forces, i.e., $F_{i}$ with $i=1,2,3$, in the Cartesian coordinates. The derivatives, $F_{i i}=\frac{\partial F_{i}}{\partial x_{i}}$, can be written as

$$
\begin{aligned}
F_{i i}= & -\left[u_{\mathrm{DD}}\left(\frac{x_{i}}{r}\right)^{2}+u_{\mathrm{D}} T_{7}-\left(\frac{x_{i}}{r}\right)^{2} T_{1} T_{5}-T_{3}\left(-\frac{2 x_{i}^{2}}{r}+T_{1} T_{7}\right)\right. \\
& +\frac{v_{r}}{r^{2}}+\frac{2 x_{i}^{2}}{r} T_{3}+3\left(\frac{x_{i}}{r}\right)^{2} T_{2} T_{6}+3 T_{4}\left(\frac{2 x_{i}^{2}}{r}+T_{2} T_{7}\right) \\
& \left.+6\left(\frac{w_{r}}{r^{2}}+\frac{x^{2}}{r} T_{4}\right)\right], \text { for } i=1,2,3 .
\end{aligned}
$$

Furthermore, the cross derivatives, $F_{i j}=\frac{\partial F_{j}}{\partial x_{i}}$ with $i \neq j$, are defined as

$$
\begin{aligned}
F_{i j}= & -\frac{x_{i} x_{j}}{r}\left[\frac{u_{\mathrm{DD}}}{r}-\frac{u_{\mathrm{D}}}{r^{2}}-\frac{T_{1}}{r} T_{5}-T_{3}\left(-2-\frac{T_{1}}{r^{2}}\right)+2 T_{3}\right. \\
& \left.+\frac{3 T_{2}}{r} T_{6}+3\left(2-\frac{T_{2}}{r^{2}}\right) T_{4}-6 T_{4}\right], \text { for } i \& j=1,2,3 .
\end{aligned}
$$

In Eqs. (A.1), (A.2), the terms $T_{1}, T_{2}, T_{3}, T_{4}, T_{5}, T_{6}$, and $T_{7}$ have following forms: $T_{1}=\left(2 x_{3}^{2}-x_{1}^{2}-x_{2}^{2}\right), T_{2}=\left(x_{1}^{2}-x_{2}^{2}\right)$, $T_{3}=\left(\frac{v_{\mathrm{D}}}{2 r^{2}}-\frac{v_{r}}{r^{3}}\right), T_{4}=\left(\frac{w_{\mathrm{D}}}{r^{2}}-\frac{2 w_{r}}{r^{3}}\right), T_{5}=\left(\frac{v_{\mathrm{DD}}}{2 r^{2}}-\frac{2 v_{\mathrm{D}}}{r^{3}}+\frac{3 v_{r}}{r^{4}}\right) T_{6}=$ $\left(\frac{w_{\mathrm{DD}}}{r^{2}}-\frac{4 w_{\mathrm{D}}}{r^{3}}+\frac{6 w_{r}}{r^{4}}\right)$, and $T_{7}=\left(\frac{1}{r}-\frac{x_{1}^{2}}{r^{3}}\right)$. Here $u_{\mathrm{DD}}, v_{\mathrm{DD}}$, and $w_{\mathrm{DD}}$ are given by

$$
\begin{aligned}
u_{\mathrm{DD}}= & \left(\frac{r}{r+1}\right)^{2-\gamma}\left(\frac{1}{r+1}-\frac{1}{r}\right)\left[\frac{2}{r+1}+(1-\gamma)\left(\frac{1}{r+1}-\frac{1}{r}\right)\right], \\
v_{\mathrm{DD}}= & -\frac{r_{1} r^{-\gamma}}{\left(r+r_{2}\right)^{4-\gamma}}\left[(1-\gamma)(2-\gamma)-2(2-\gamma)(4-\gamma) \frac{r}{\left(r+r_{2}\right)}\right. \\
& \left.+(4-\gamma)(5-\gamma) \frac{r^{2}}{\left(r+r_{2}\right)^{2}}\right], \\
w_{\mathrm{DD}}= & -\frac{r_{3} r^{-\gamma}}{\left(r+r_{4}\right)^{4-\gamma}}\left[(1-\gamma)(2-\gamma)-2(2-\gamma)(4-\gamma) \frac{r}{\left(r+r_{4}\right)}\right. \\
& \left.+(4-\gamma)(5-\gamma) \frac{r^{2}}{\left(r+r_{4}\right)^{2}}\right],
\end{aligned}
$$

which represent the derivatives of $u_{\mathrm{D}}, v_{\mathrm{D}}$, and $w_{\mathrm{D}}$ with respect to $r$, respectively.

\section{References}

Benettin, G., Galgani, L., Giorgilli, A., \& Strelcyn, J.-M. 1980, Meccanica, 15,21 Binney, J. J. 1978, Comm. Astrophys., 8, 27

Chakraborty, D. K., \& Thakur, P. 2000, MNRAS, 318, 1273

Crane, P., Stiavelli, S., King, I. R., et al. 1993, AJ, 106, 1371

Dehnen, W. 1993, MNRAS, 265, 250

de Zeeuw, P. T., \& Carollo, C. M. 1996, MNRAS, 281, 1333 (ZC96)

Faber, S. M., Tremaine, S. Ajhar, E. A., et al. 1997, AJ, 114, 1771

Fehlberg, E. 1968, NASA Tech. Rep. TR R-287

Ferrarese, L., van den Bosch, F. C., Ford, H. C., Jaffe, W., \& O’Connell, R. W. 1994, AJ, 108, 1598

Holley-Bockelmann, K., Mihos, J. C., Sigurdsson, S., \& Hernquist, L. 2001, ApJ, 549,862

Holley-Bockelmann, K., Mihos, J. C., Sigurdsson, S., \& Hernquist, L. 2002, ApJ, 567,817

Jaffe, W., Ford, H. C., O’Connell, R. W., van den Bosch, F. C., \& Ferrarese, L. 1994, AJ, 108, 1567

Jedrzejewski, R. I. 1987, ed. P. T. de Zeeuw, Proc. IAU Symp., 127, Structure and Dynamics of Elliptical Galaxies (Dordrecht: Reidel), 37

Kandrup, H. E., \& Siopis, C. 2003, MNRAS, 345, 727

Lauer, T., et al. 1995, AJ, 110, 2622

Lucy, L. B. 1974, AJ, 79, 745

Merritt, D., \& Fridman, T. 1996 ApJ, 460, 136

Merritt, D., \& Valluri, M. 1996, ApJ, 471, 82

Moller, P., Staivelli, M., \& Zeilinger, W. W. 1995, MNRAS, 276, 979

Peletier, R. F., Davis, R. L., Illingworth, G. D., Davis, L. E., \& Cawson, H. 1990, AJ, 100, 1091

Poon, M. Y., \& Merritt, D. 2004, ApJ, 606, 774

Schwarzschild, M. 1979, ApJ, 232, 236

Schwarzschild, M. 1993, ApJ, 409, 563

Siopis, C., \& Kandrup, H. E. 2000, MNRAS, 319, 43

Thakur, P., \& Chakraborty, D. K. 2001, MNRAS, 328, 330

Udry, S., \& Pfenniger, D. 1988, A\&A, 198, 135

Wachlin, F. C., \& Ferraz-Mello, S. 1998, MNRAS, 298, 22 